\title{
Determinación de la Velocidad de Transporte de Partículas por Saltos en Conductos Horizontales
}

\author{
Jorge E. Flores ${ }^{(1,2)}$, Gloria Villaflor ${ }^{(1,2,3)}$, Jorge W. Velasco ${ }^{(1,2,3)}$ y Eduardo R. Bisonard ${ }^{(1,2)}$ \\ (1) Instituto de Beneficio de Minerales, Facultad de Ingeniería, Universidad Nacional de Salta, \\ Avda. Bolivia 5150, 4400, Salta, Argentina \\ (2) Consejo de Investigación, Universidad Nacional de Salta, CIUNSa., Avda. Bolivia 5150, \\ 4400, Salta, Argentina. \\ (3) INIQUI. CONICET, Avda. Bolivia 5150, 4400, Salta, Argentina. (e-mail: jeflores@unsa.edu.ar; \\ villaflo@unsa.edu.ar)
}

Recibido Abr. 28, 2017; Aceptado Jul. 11, 2017; Versión final Ago. 28, 2017, Publicado Dic. 2017

\begin{abstract}
Resumen
Se determina experimentalmente la velocidad mínima de transporte para diferentes materiales y tamaños de partículas y dos diámetros de conducto. Para reducir la erosión de los conductos y el consumo energético del equipo aspirador, es conveniente que la velocidad de flujo del aire sea la menor posible, siendo este valor la velocidad de transporte por saltos de las partículas. La literatura técnica proporciona valores de dicha velocidad mínima del aire. Se verifican los valores experimentales con los calculados utilizando las correlaciones mencionadas y se estudia la influencia del tamaño del conducto en el cálculo de la velocidad de transporte por saltos. Este estudio muestra que la variación del diámetro del conducto tiene poca influencia en la velocidad de transporte por saltos para las situaciones estudiadas en este trabajo.
\end{abstract}

Palabras claves: ventilación localizada; transporte de partículas; velocidad mínima de aire; velocidad de saltos

\section{Determination of the Velocity of ParticlesTransported by Saltation in Horizontal Ducts}

\begin{abstract}
The minimum transport velocity for different materials and particle sizes is determined experimentally for two duct diameters. To reduce the erosion of such ducts and the energy consumption of the aspirating device, it is advantageous that the design velocity of the air flow be the lowest possible, being this the transport saltation velocity of the particles. The technical literature provides values of this minimum air velocity. The experimental values are verified with those calculated using the aforementioned correlations and the influence of the duct size in the calculation of the transport saltation velocity is studied. This study shows that the variation of the diameter of the duct has little influence on the variation of the transport velocity by saltation for the experimentally conditions studied in this work.
\end{abstract}




\section{INTRODUCCIÓN}

Las instalaciones de ventilación localizada se utilizan en plantas industriales para captar y transportar el polvo generado en las distintas operaciones que involucran materiales sólidos, como por ejemplo, trituración, molienda, clasificación, transporte y almacenamiento (Patankar et al., 2017; Cabrejos et al., 2004; Jo y Ray, 1999). Estas instalaciones son importantes para cumplir con objetivos específicos de la higiene y seguridad industrial. Asimismo en la industria se transporta un amplio rango de sólidos particulados utilizando transporte neumático, desde carbón, cemento, harina o granos de cereales a pellets de alimentos balanceados. Los sistemas de transporte neumático de partículas en fase diluida se caracterizan por altas velocidades de aire, bajas concentraciones de sólidos y bajas pérdidas de carga por unidad de longitud de cañería, siendo los más usados en la industria porque son más estables y permiten mayor flujo y mayores distancias de transporte, como señalan Setia et al. (2015) y Cabrejos et al (2004).

En estos sistemas es necesario determinar la velocidad mínima de transporte de las partículas en los conductos para evitar la sedimentación de las mismas (Deng y Bradley, 2016). Por razones de seguridad operativa es conveniente que la velocidad de diseño sea mayor que dicha velocidad mínima para impedir el taponamiento del conducto (Hartman et al., 2006), la sedimentación de sólidos con un cierto contenido de humedad o de partículas aglomeradas por efectos electrostáticos y permitir el transporte del sólido que podría haber sedimentado debido a una incorrecta operación del sistema (Pocoví, 1999). La deposición de sólido puede formar un lecho en la parte inferior del conducto, reduciendo su sección transversal (Jo y Ray, 1999). Sin embargo, si la velocidad de diseño es excesiva puede producir la erosión de los conductos, el desgaste de las partículas y fundamentalmente el aumento del consumo energético del ventilador requerido (Rabinovich y Kalman, 2011). De esta forma, el diseño de las instalaciones de ventilación localizada, es importante no solamente desde el punto de vista de la higiene y seguridad industrial, sino también por el aspecto económico y energético, porque seleccionar una velocidad elevada en el conducto puede llevar a instalaciones sobredimensionadas, con el consecuente aumento de la energía consumida.

La definición de velocidad mínima de transporte utilizada en la literatura técnica no es precisa, ya que pueden determinarse dos velocidades mínimas, correspondientes a los tramos de conductos verticales y a los horizontales, debido a que el mecanismo de sedimentación del sólido es diferente en cada caso. En los conductos horizontales pueden definirse además, otras velocidades relacionadas con la velocidad mínima como son las velocidades de transporte por saltos y la velocidad de arranque o pickup (Khan et al., 2016; Rabinovich y Kalman, 2011; Hubert y Kalman, 2003; Cabrejos y Klinzing, 1994). En los conductos verticales la sedimentación del sólido comienza cuando la velocidad del aire disminuye hasta igualar la velocidad terminal de sedimentación de las partículas sólidas. En cambio, en los conductos horizontales la sedimentación es más compleja debido a que la fuerza de gravedad, o sea el peso de las partículas no coincide con la dirección en que tiene lugar el transporte. Si en estos conductos se va disminuyendo la velocidad de flujo de aire, partiendo de valores superiores al mínimo requerido para el transporte, se observa que se alcanza lo que se denomina velocidad crítica de transporte. Las trayectorias de las partículas dejan de ser continuas y se transforman en saltos en la parte inferior del conducto, esto es lo que se denomina velocidad de transporte por saltos (Khan et al., 2016; Pocoví, 1999).

Tanto en las instalaciones de ventilación como las de transporte neumático, por razones de seguridad operativa es conveniente que la velocidad de diseño del conducto sea mayor que la velocidad mínima de transporte o sea que la velocidad mínima alcance a ser por lo menos igual a la de transporte por saltos. Como las instalaciones de ventilación localizada captan normalmente partículas de granulometría fina, este tipo de instalaciones pueden ser diseñadas sobre la base de considerar que la velocidad mínima de transporte es igual a la velocidad de transporte por saltos. En la literatura técnica se encuentran propuestas distintas correlaciones semiteóricas y empíricas que permiten calcular la velocidad de transporte de partículas sólidas en conductos horizontales en función del tamaño y densidad de las partículas, de la relación sólido/aire y en algunos casos de la densidad del aire y del diámetro del tubo. El Manual de Ventilación Industrial, de la American Conference of Governmental Industrial Hygienists (ACGIH, 1995), especifica valores de la velocidad mínima de flujo de aire para evitar la sedimentación de las partículas sólidas transportadas. Estos valores de velocidad varían en función de la naturaleza del material transportado, siendo los rangos de velocidades recomendados no tan precisos y no se dan en forma específica la relación de dichas velocidades con las propiedades y características de las partículas. Esto puede llevar en muchos casos al diseño de instalaciones con velocidades mayores a las requeridas, con el consiguiente aumento del consumo energético.

\section{VELOCIDAD DE TRANSPORTE DE PARTÍCULAS}

Se analizan las correlaciones de Dalla Valle (Anaya Durand et al., 2002; Jones y Leung, 1978), Pocoví (1999), Zenz (Matsumoto et al., 1974; Leva, 1959), Cabrejos-Klinzing (Gómes y Amarante Mesquita, 2014; Cabrejos y Klinzing, 1994), Molerus (Molerus y Heucke, 1999), Rizk, Rose y Duckworth (Jones y Leung, 1978), Schade 
(Gómes y Amarante Mesquita, 2014; Hubert y Kalman, 2003) y Matsumoto (Matsumoto et al., 1975). En general, dichas correlaciones están expresadas en unidades no homogéneas. En este trabajo se trata de conservar la nomenclatura utilizada por cada autor.

En la Tabla 1 se muestran las correlaciones de Dalla Valle y de Pocoví para calcular la velocidad mínima de transporte, igual a la velocidad de transporte por saltos. Estas correlaciones dependen de las propiedades y características de las partículas, densidad y tamaño, pero no tienen en cuenta la concentración de sólidos, que puede ser considerada como muy importante (Jones y Leung, 1978). Sin embargo, para ventilación localizada los valores de la relación sólido/aire son bajos, disminuyendo su importancia en el cálculo de la velocidad (Hubert y Kalman, 2003). La correlación de Pocoví es válida para partículas de granulometría fina, $\mathrm{dp}<0,5 \mathrm{~mm}$, tamaños normales para instalaciones de ventilación localizada. Asimismo el autor recomienda utilizar para el diseño de las instalaciones un factor de seguridad del orden del 50 al 100\%, para tener en cuenta la humedad del sólido o efectos electrostáticos que tienden a aglomerar las partículas (Pocoví, 1999).

Tabla 1: Velocidad por saltos en función de la densidad y tamaño de las partículas

\begin{tabular}{|l|c|c|c|c|}
\hline \multirow{2}{*}{ Correlación } & \multirow{2}{*}{$\begin{array}{c}\text { Velocidad, } \\
\mathrm{m} / \mathrm{s}\end{array}$} & $\begin{array}{c}\text { Unidades } \\
\text { Densidad del } \\
\text { sólido, } \rho_{\mathrm{s}}\end{array}$ & $\begin{array}{c}\text { Diámetro de } \\
\text { partícula, } d_{p}\end{array}$ & \multirow{2}{*}{ Constantes } \\
\hline Dalla Valle & $\mathrm{v}_{\mathrm{s}}=\mathrm{k} \frac{\rho_{\mathrm{s}}}{\left(\rho_{\mathrm{s}}+1000\right)} \mathrm{dp}$ & $\mathrm{kg} / \mathrm{m}^{3}$ & $\mathrm{~mm}$ & $\begin{array}{c}\mathrm{k}=8,35 \\
\mathrm{n}=0,4\end{array}$ \\
\hline Pocoví & $\mathrm{v}_{\min }=5,4 \rho_{\mathrm{s}}^{0,37} \mathrm{~d}_{\mathrm{p}}^{0,26}$ & $\mathrm{~g} / \mathrm{cm}^{3}$ & $\mathrm{~mm}$ & 5,4 \\
\hline
\end{tabular}

En la Tabla 2 se muestran las correlaciones propuestas por Zenz, Cabrejos-Klinzing y Molerus para calcular la velocidad de transporte por saltos teniendo en cuenta la relación sólido/aire. Zenz calcula la velocidad de saltos a partir de $u_{s}=\rho_{s} \sqrt{k g_{c} d_{p}}$, donde $k$ es una constante adimensional para cada valor de relación sólido/aire, $\mathrm{m}_{\mathrm{s}}, \mathrm{y} \mathrm{g}_{\mathrm{c}}\left(\mathrm{lb} \mathrm{ft} / \mathrm{lbf} \mathrm{s}^{2}\right.$ ) es un factor de conversión para consistencia de unidades. Realizando la regresión de los datos experimentales publicados por Zenz (Leva, 1959), se obtiene $\mathrm{k}=0,04 \mathrm{e}^{0,132 \mathrm{~ms}}$.

Tabla 2: Velocidad por saltos en función de la densidad y tamaño de las partículas y/o la relación sólido/aire

\begin{tabular}{|c|c|c|c|c|c|}
\hline \multirow[b]{2}{*}{ Correlación } & \multirow[b]{2}{*}{ Velocidad } & \multicolumn{3}{|c|}{ Unidades } & \multirow{2}{*}{ Ctes. } \\
\hline & & Velocidad & Densidad & $\begin{array}{c}\text { Diámetro/ } \\
\text { Caudal sólido }\end{array}$ & \\
\hline Zenz & $u_{s}=\sqrt{g_{c} d_{p} \rho_{s}^{2} 0,04 e^{0,132 m_{s}}}$ & $\mathrm{ft} / \mathrm{s}$ & $\rho_{\mathrm{s}}: \mathrm{lb} / \mathrm{ft}^{3}$ & $\begin{array}{l}\mathrm{dp}_{\mathrm{p}}: \mathrm{ft} \\
\mathrm{ms}_{\mathrm{s}}: \operatorname{adim} .\end{array}$ & $g_{c}=32,2$ \\
\hline $\begin{array}{l}\text { Cabrejos- } \\
\text { Klinzing }\end{array}$ & $u_{g s}=\sqrt{g_{p}} 0,000224\left(\frac{\rho_{p}}{\rho_{g}}\right)^{1,25} \mu^{0.25}$ & $\mathrm{~m} / \mathrm{s}$ & $\begin{array}{l}\rho_{p}: \mathrm{kg} / \mathrm{m}^{3} \\
\rho_{\mathrm{g}}: \mathrm{kg} / \mathrm{m}^{3}\end{array}$ & $\begin{array}{l}\mu \text { : relación } \\
\text { sólido/aire, } \\
\text { adim. }\end{array}$ & $\begin{array}{l}\mathrm{g}=9,81 \\
\mathrm{~m} / \mathrm{s}^{2}\end{array}$ \\
\hline Molerus & $v_{f}=5 \sqrt[1]{\frac{M_{s} g^{2}}{\rho_{f}}}$ & $\mathrm{~m} / \mathrm{s}$ & $\rho_{f}: \mathrm{kg} / \mathrm{m}^{3}$ & $M_{s}: \mathrm{kg} / \mathrm{s}$ & $\begin{array}{l}g=9,81 \\
\mathrm{~m} / \mathrm{s}^{2}\end{array}$ \\
\hline
\end{tabular}

En la Tabla 3 se muestran correlaciones propuestas por Rizk, Rose y Duckworth, Schade y Matsumoto para calcular la velocidad de transporte por saltos. Estas correlaciones tienen en cuenta diámetro interno de la cañería, D, además de la relación sólido/aire, la densidad y el diámetro de las partículas y la densidad del aire en alguna de ellas. Rizk calcula la velocidad de saltos a partir de la relación sólido/aire, $\mathrm{m}_{\mathrm{s}}=\left(1 / 10^{\delta}\right) \mathrm{Fr}_{\mathrm{s}}{ }^{\mathrm{N}}$ y Froude de salto, $\mathrm{Fr}_{\mathrm{s}}=\mathrm{v}_{\mathrm{s}} / \sqrt{\mathrm{gD}}$, siendo $\delta$ y $\mathrm{N}$ funciones del diámetro de partículas. Rose and Duckworth recomiendan tomar la velocidad del aire de dos a tres veces la obtenida con dicha ecuación.

A partir de la correlación propuesta por Matsumoto (1975), $m_{s}=1,11\left(\rho_{p} / \rho_{s}\right)^{0,55}\left(F_{p} / 10\right)^{-2,3}\left(F_{s} / 10\right)^{3,0}$, se obtiene la expresión de la velocidad de transporte por saltos, siendo $\mathrm{Fr}_{p}$ el número de Froude de partícula, $\mathrm{Fr}_{\mathrm{p}}=\mathrm{u}_{\mathrm{t}} / \sqrt{\mathrm{gd}_{\mathrm{p}}}$ y $\mathrm{Fr}_{\mathrm{s}}$ el número de Froude de salto, $\mathrm{Fr}_{\mathrm{s}}=\mathrm{u}_{\mathrm{s}} / \sqrt{\mathrm{gd}_{\mathrm{p}}}$. Los rangos de aplicación recomendados por el autor corresponden a tamaños y densidades de sólidos comprendidos entre 0,29 $\mathrm{mm}<\mathrm{d}_{\mathrm{p}}<2,6 \mathrm{~mm}$ y $1,0 \mathrm{~g} / \mathrm{cm}^{3}<\rho_{p}<8,7 \mathrm{~g} / \mathrm{cm}^{3}$, respectivamente (Matsumoto et al., 1975, Matsumoto et al., 1974). 
Tabla 3: Velocidad por saltos en función del tamaño de las partículas, la densidad y la relación sólido/aire

\begin{tabular}{|c|c|c|}
\hline Correlación & Velocidad, $\mathrm{m} / \mathrm{s}$ & Unidades y constantes \\
\hline Rizk & $\mathrm{v}_{\mathrm{s}}=\sqrt{\mathrm{g} \mathrm{D}}\left(\mathrm{m}_{\mathrm{s}} 100^{\left.1440 \mathrm{~d}_{\mathrm{p}}+1,96\right) \frac{1}{1100 \mathrm{~d}_{\mathrm{p}}+2,5}}\right.$ & \multirow{4}{*}{$\begin{array}{l}\text { Densidades } \\
\rho_{\mathrm{p}}, \rho_{\mathrm{s}}: \mathrm{kg} / \mathrm{m}^{3} \\
\rho_{\mathrm{a}}, \rho_{\mathrm{g}}: \mathrm{kg} / \mathrm{m}^{3} \\
\text { Diámetro de partículas, } d_{\mathrm{p}}: \mathrm{m} \\
\text { Diámetro interno de cañería, D: } \mathrm{m} \\
\text { Constantes: } \mathrm{g}=9,81 \mathrm{~m} / \mathrm{s}^{2}\end{array}$} \\
\hline Rose and Duckworth & $u_{s}=\left[v_{t} 3,2 m_{s}^{0,2}\left(\frac{D}{d_{p}}\right)^{0,6}\left(\frac{\rho_{s}}{\rho_{g}}\right)^{-0,7}(g D)^{-0,25}\right]^{2}$ & \\
\hline Schade & $u_{s}=(g \text { D })^{0,5} m_{s}^{0,11}\left(\frac{D}{d_{p}}\right)^{0,025}\left(\frac{\rho_{p}}{\rho_{g}}\right)^{0,34}$ & \\
\hline Matsumoto & $u_{s}=\left(\left(\frac{\rho_{a}}{\rho_{p}}\right)^{0,5} g^{0,65} d_{p}^{-0,875} D^{1,5} 10^{1,25} \frac{m_{s}}{0,448}\right)^{\frac{1}{1,25}}$ & \\
\hline
\end{tabular}

En la Tabla 4 se indican los rangos de valores de velocidades de diseño para las instalaciones de ventilación industrial localizada recomendados por la American Conference of Governmental Industrial Hygienists (ACGIH, 1995). Estos valores dependen del tipo de partículas, tamaño (muy finas, finas o gruesas), densidad (muy baja, baja, media o alta) y humedad (secas o húmedas).

Tabla 4: Rango de velocidades de diseño recomendadas por ACGIH

\begin{tabular}{|l|c|}
\hline Tipo de partículas & Velocidades de diseño $(\mathrm{m} / \mathrm{s})$ \\
\hline Humos metalúrgicos & 7 a 10 \\
\hline Muy finas y de muy baja densidad aparente & 10 a 13 \\
\hline Finas y secas de materiales de baja densidad & 13 a 18 \\
\hline Densidad media o baja, húmedas & 18 a 20 \\
\hline Gruesas de alta densidad & 20 a 23 \\
\hline Muy alta densidad o húmedas & $>23$ \\
\hline
\end{tabular}

\section{TRABAJO EXPERIMENTAL}

El equipo experimental utilizado para las determinaciones de la velocidad de transporte se muestra en la Fig. 1. Consta de un conducto horizontal de acrílico transparente y permite intercambiar dos tubos de distinto diámetro de las siguientes dimensiones: uno de $82,5 \mathrm{~mm}$ de diámetro, $2540 \mathrm{~mm}$ de largo y $3 \mathrm{~mm}$ de espesor y el otro de $70 \mathrm{~mm}$ de diámetro, $2555 \mathrm{~mm}$ de largo y $3 \mathrm{~mm}$ de espesor.

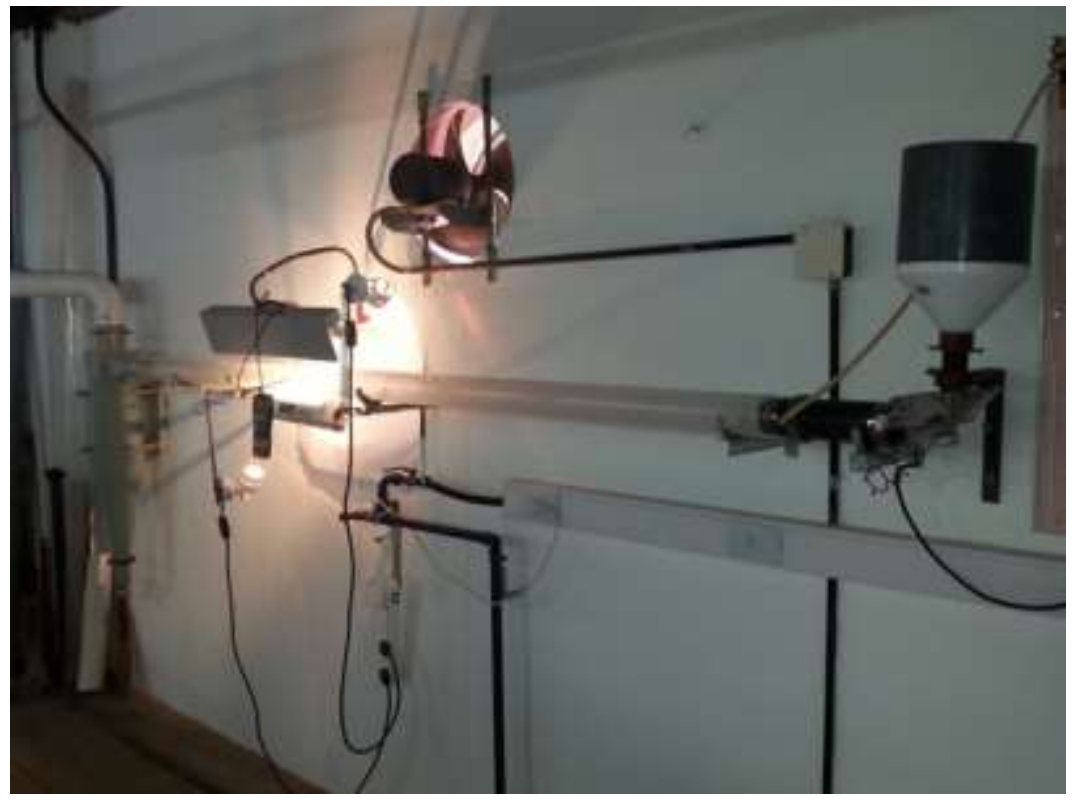

Fig. 1: Equipo experimental para la determinación de la velocidad de transporte por saltos 
El material que se transporta es alimentado en forma continua por medio de una tolva con descarga vibratoria mediante un sistema electromagnético, que permite variar los valores de la relación sólido/aire y es recuperado mediante un ciclón de alta eficiencia (Pocoví et al., 2002; Pocoví et al., 1998) de $200 \mathrm{~mm}$ de diámetro. La aspiración del caudal de aire requerido se logra por medio de un ventilador centrífugo, accionado por un motor eléctrico de $4 \mathrm{Hp}$ de $2800 \mathrm{rpm}$ y regulado por un variador de frecuencia. La velocidad de flujo del aire se mide por medio de un anemómetro compacto Testo 425, con sonda de velocidad y temperatura integrada y empuñadura telescópica. Para apreciar mejor el movimiento de las partículas, una parte del conducto de acrílico está iluminado por un tubo de 30 luces led y 2 lámparas dicroicas, que otorgan una clara visión del interior del tubo.

\section{PROGRAMA DE CÁLCULO}

Se realizó una Planilla de Cálculo en Excel para determinar las velocidades de transporte por saltos utilizando las correlaciones propuestas en la literatura técnica por Dalla Valle, Pocoví, Zenz, Cabrejos-Klinzing, Molerus, Rizk, Rose y Duckworth, Schade y Matsumoto, en función de la densidad, granulometría y concentración de sólidos de cada material, densidad del aire y diámetro del conducto, según corresponda. Para calcular el diámetro de las partículas, si la granulometría es estrecha, se toma como diámetro medio la media aritmética de las aberturas de malla correspondiente a los dos tamices estándar consecutivos que caracterizan la fracción granulométrica. Si la granulometría del sólido es amplia, lo que es frecuente en la práctica, deberá tomarse como diámetro medio la fracción granulométrica estrecha más gruesa presente. Se calcula además la potencia del ventilador que es función del caudal de aire, de las caídas de presión y de la eficiencia mecánica del mismo. Es conveniente por lo tanto que la velocidad de diseño del flujo de aire sea la menor posible para reducir el consumo energético del equipo de aspiración.

\section{RESULTADOS Y DISCUSIÓN}

Se realizaron ensayos experimentales por triplicado utilizando arena, colemanita y mineral de manganeso, con fracciones estrechas de tamaño correspondientes a mallas ASTM: 30-50 M, 50- 70 M y 70-100 M. Estos ensayos fueron realizados utilizando dos tubos distintos, de $82,5 \mathrm{~mm}$ y de $70 \mathrm{~mm}$ de diámetro, respectivamente. Los ensayos se llevaron a cabo con valores bajos de la relación sólido/aire, tales como los utilizados en ventilación localizada. Se trabajó con relaciones sólido/aire $(p / p)$ iguales a 0,$11 ; 0,23 ; 0,36$ y 0,45 . Los ensayos se realizaron comenzando con altas velocidades de flujo, las que progresivamente se fueron disminuyendo mediante el regulador de frecuencia, hasta que se observó que el material empezó a ser transportado por saltos, registrando en ese momento su velocidad (Hubert y Kalman, 2003). En la Fig. 2 se muestra el transporte de partículas por saltos.

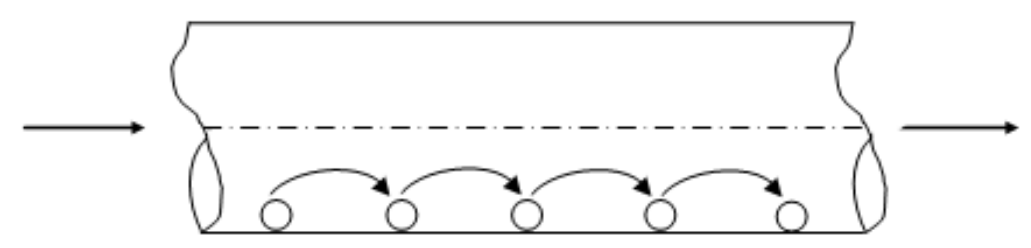

Fig. 2: Transporte de partículas por saltos

Utilizando la Planilla de Cálculo desarrollada se determinaron las velocidades de transporte por saltos con las correlaciones propuestas en la literatura técnica y se compararon con los valores obtenidos experimentalmente y los recomendados por American Conference of Governmental Industrial Hygienists en Industrial Ventilation. En las Tablas 5 y 6 se muestran los resultados experimentales obtenidos de la velocidad de transporte por saltos, la densidad y el diámetro medio de partícula, para los materiales y tamaños estudiados, para una relación sólido/aire de 0,11.

Tabla 5: Resultados experimentales de la velocidad de transporte por saltos para un diámetro de $82,5 \mathrm{~mm}$

\begin{tabular}{|l|c|c|c|c|}
\hline \multicolumn{1}{|c|}{ Material } & Densidad $\mathrm{g} / \mathrm{cm}^{3}$ & Fracción $(\mathrm{M})$ & $d p(\mathrm{~mm})$ & $v_{t s}(\mathrm{~m} / \mathrm{s})$ \\
\hline \multirow{3}{*}{ Arena } & \multirow{2}{*}{2,5} & $30-50$ & 0,446 & 4,18 \\
\cline { 3 - 5 } & & $50-70$ & 0,254 & 3,94 \\
\cline { 3 - 5 } & & $70-100$ & 0,18 & 3,80 \\
\hline \multirow{3}{*}{ Colemanita } & \multirow{2}{*}{2,1} & $30-50$ & 0,446 & 4,18 \\
\cline { 3 - 5 } & & $50-70$ & 0,254 & 3,71 \\
\cline { 3 - 5 } & & $70-100$ & 0,18 & 3,48 \\
\hline \multirow{2}{*}{ Mineral de Manganeso } & \multirow{3}{*}{4,8} & $30-50$ & 0,446 & 5,34 \\
\cline { 3 - 5 } & & $50-70$ & 0,254 & 4,87 \\
\cline { 3 - 5 } & & $70-100$ & 0,18 & 4,41 \\
\hline
\end{tabular}


Tabla 6: Resultados experimentales de la velocidad de transporte por saltos para un diámetro de $70 \mathrm{~mm}$

\begin{tabular}{|c|c|c|c|c|}
\hline Material & $\begin{array}{c}\text { Densidad } \\
\mathrm{g} / \mathrm{cm}^{3}\end{array}$ & $\begin{array}{c}\text { Fracción } \\
M\end{array}$ & $\begin{array}{c}d p \\
\mathrm{~mm}\end{array}$ & $\begin{array}{c}v_{t s} \\
\mathrm{~m} / \mathrm{s}\end{array}$ \\
\hline \multirow{3}{*}{ Arena } & \multirow{3}{*}{2,5} & $30-50$ & 0,446 & 4,00 \\
\cline { 3 - 5 } & & $50-70$ & 0,254 & 3,52 \\
\cline { 3 - 5 } Colemanita & \multirow{3}{*}{2,1} & $70-100$ & 0,18 & 3,20 \\
\cline { 3 - 5 } & & $30-50$ & 0,446 & 3,93 \\
\cline { 3 - 5 } & & $50-70$ & 0,254 & 3,50 \\
\cline { 3 - 5 } & & $70-100$ & 0,18 & 3,33 \\
\hline
\end{tabular}

En las Figuras 3 a 7 se representa la velocidad de transporte por saltos en función del diámetro de partícula para arena, colemanita y mineral de manganeso. Se representan los valores determinados experimentalmente con los dos tamaños de tubo y los obtenidos con la Planilla de cálculo aplicando las correlaciones mencionadas.

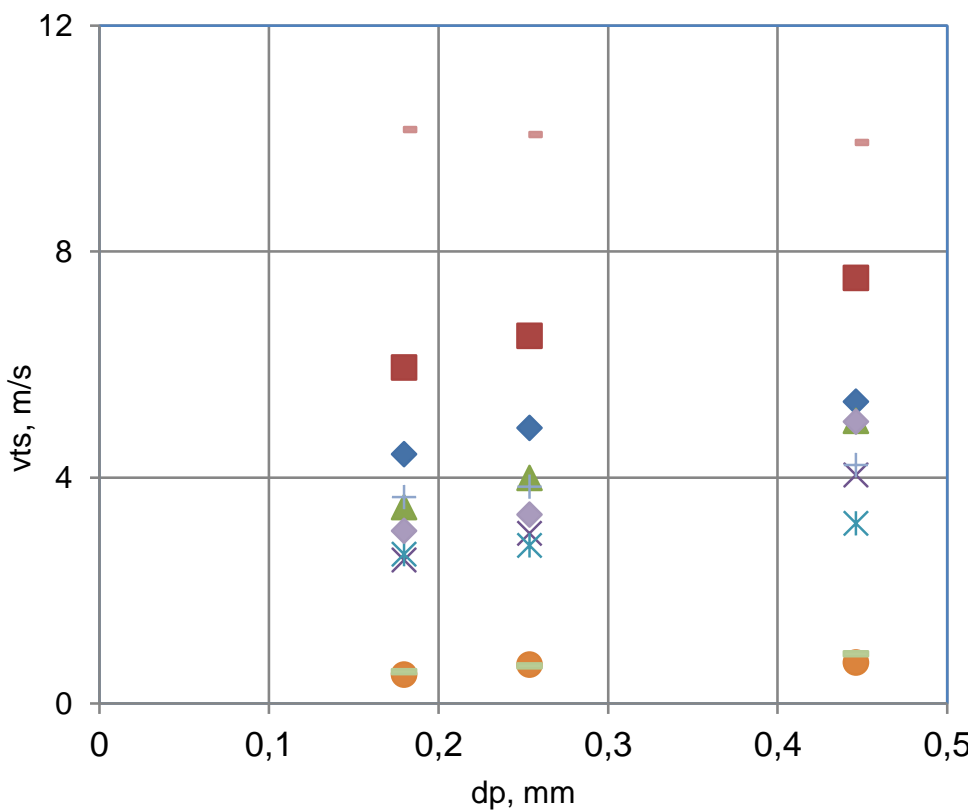

$\checkmark$ Veloc. de saltos experimentales

- Correl. de Pocoví

$\triangle$ Correl. de Dalla Valle

$\times$ Correl. de Zenz

* Correl. de Risk

- Correl. de Rose and Duckworth

+ Correl. de Molerus

- Correl. de Schade

- Correl. de Cabrejos-Klinzing

Correl. de Matsumoto

Fig. 3: Velocidad de transporte de saltos para manganeso y diámetro de conducto de $82,5 \mathrm{~mm}$

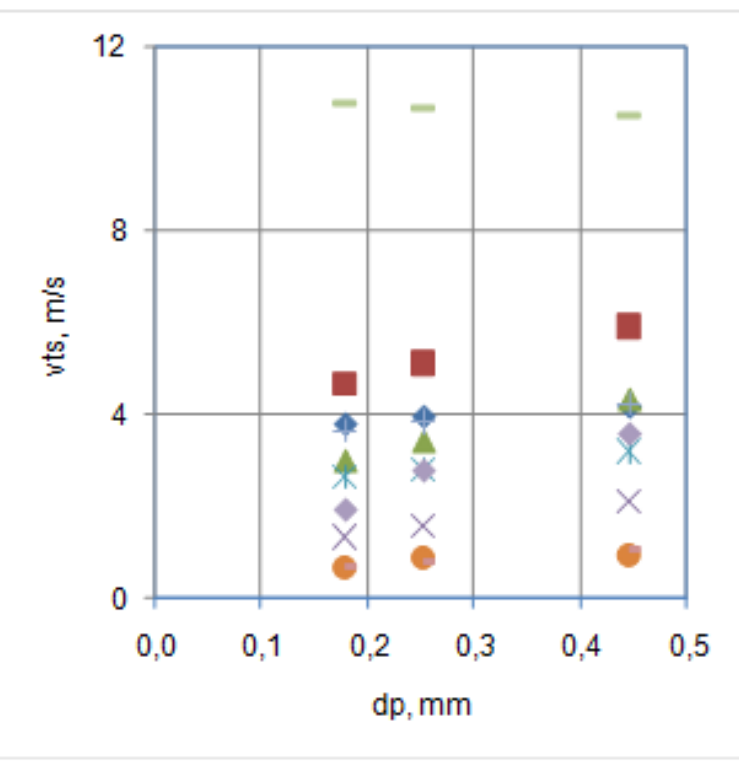

Fig. 4: Velocidad de transporte de saltos para arena y diámetro de conducto de $82,5 \mathrm{~m}$

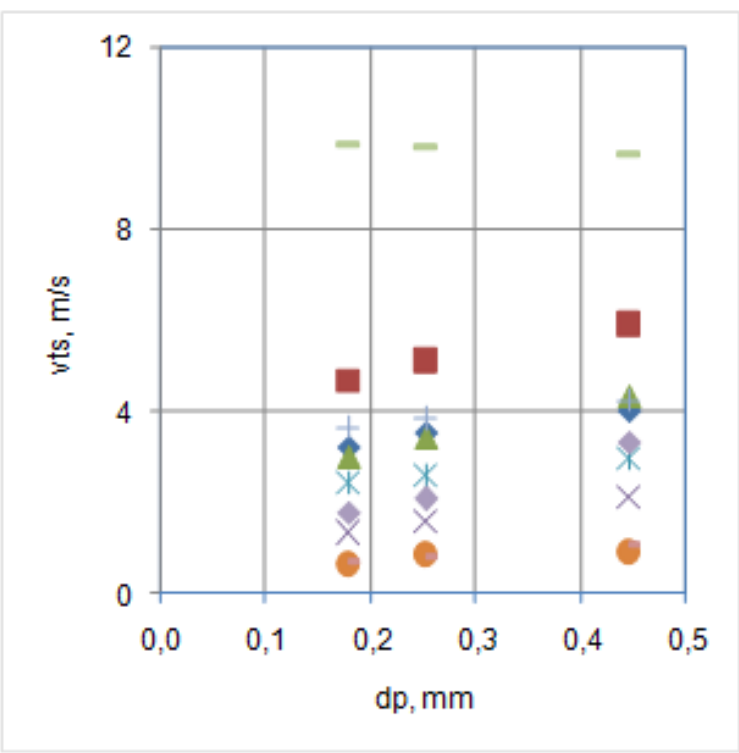

Fig. 5: Velocidad de transporte de saltos para arena y diámetro del conducto de $70 \mathrm{~mm}$ 


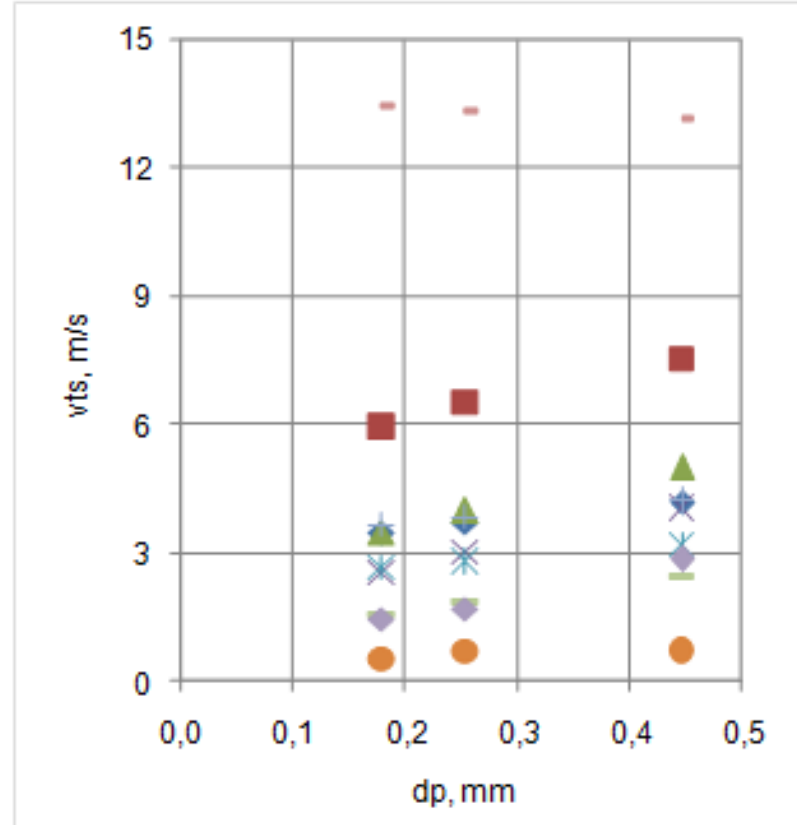

Fig. 6: Velocidad de transporte por saltos para colemanita y diámetro de conducto de $82,5 \mathrm{~mm}$

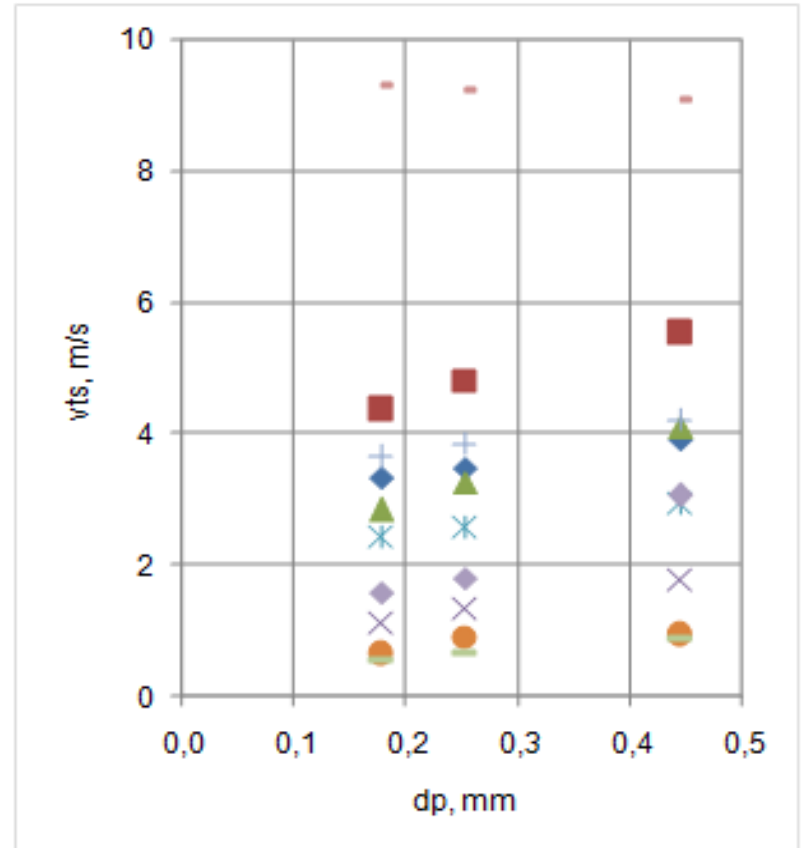

Fig. 7: Velocidad de transporte por saltos para colemanita y diámetro de conducto de $70 \mathrm{~mm}$

Los valores obtenidos, para relación sólido/aire igual a 0,11 , aplicando las correlaciones de Pocoví, Dalla Valle, Rizk, Molerus y Matsumoto difieren en menos del $50 \%$ con respecto a los valores experimentales. La correlación de Zenz difiere en valores mayores al $50 \%$ y las de Rose and Duckworth y Cabrejos-Klinzing en alrededor del $80 \%$. En cambio la correlación de Schade difiere en más del $100 \%$, razón por la cual no se aconseja su uso para estas condiciones (Khan et al., 2016). Además, a diferencia de las otras correlaciones, que están en función del diámetro del conducto, la correlación de Schade parece mostrar que la velocidad de saltos disminuye con el aumento del tamaño de partícula, lo que tampoco ocurre con los datos obtenidos experimentalmente. La correlación de Zenz, salvo para el caso de mineral de manganeso donde da valores similares a la de Risk, se aleja de los valores obtenidos experimentalmente.

Las correlaciones de Dalla Valle, Pocoví, Rizk, Molerus y Matsumoto se aproximan mucho más a los valores obtenidos experimentalmente que los indicados por el Manual de Ventilación Industrial para el cálculo de la velocidad de diseño en los conductos de ventilación. Se considera que la razón de esta diferencia se debe, evidentemente, a la aplicación de un factor de seguridad alto por dicho Manual de Ventilación, del orden de 3 a 3,5. En la elección del factor de seguridad se debe tener cuidado, ya que un alto valor del factor significa un incremento elevado de la velocidad de flujo y un aumento de la potencia consumida por el ventilador. Aumentando las relaciones sólido/aire se observa que las correlaciones de Risk y de Matsumoto predicen mejor los valores experimentales, siendo éstas las más recomendadas para transporte neumático de sólidos en fase diluida y para tamaños mayores de partículas, la que mejor correlaciona es la de Matsumoto. Con respecto a la variación del diámetro del conducto, los datos experimentales y las correlaciones estudiadas muestran muy poca influencia en la variación de la velocidad de transporte de saltos con el aumento del mismo.

\section{CONCLUSIONES}

De acuerdo a los resultados de este estudio se pueden extraer las siguientes conclusiones: 1) Los valores de velocidad determinados experimentalmente para los materiales y tamaños utilizados, pueden ser mejor representados por las correlaciones de Dalla Valle, Pocoví, Rizk, Molerus y Matsumoto. La velocidad predicha por la correlación de Matsumoto se aproxima más a los valores experimentales a medida que aumenta la relación sólido/aire y el diámetro de las partículas. 2) La correlación de Schade es la que más se aleja de los valores experimentales, lo que llevaría a no considerar su aplicación para las condiciones utilizadas en este trabajo. 3) La variación del diámetro del conducto muestra poca influencia en la variación de la velocidad de transporte por saltos para los valores obtenidos experimentalmente. Asimismo, las correlaciones consideradas, que tienen en cuenta el diámetro del conducto, principalmente las de Matsumoto y Risk, presentan también escasa influencia. 4) Los valores obtenidos con las correlaciones usadas se aproximan mucho más a los valores experimentales que los indicados por el Manual Industrial Ventilation, permitiendo así trabajar con velocidades menores y disminuyendo consecuentemente el consumo energético del ventilador. 


\section{REFERENCIAS}

American Conference of Governmental Industrial Hygienists, Industrial Ventilation. A Manual of Recommended Practice, 22 ${ }^{\text {nd }}$. Ed., ACGIH, USA (1995)

Anaya Durand, A., E. G. Santamaría Rodríguez y K. M. Martínez Pérez, Flujo de Dos Fases con Materiales Sólidos, Ingeniería Química, XXXIV, (387), 91-98 (2002)

Cabrejos, F. y G. E. Klinzing, Minimum Conveying Velocity in Horizontal Pneumatic Transport and the Pickup and Saltation Mechanisms of Solid Particles, Bulk Solids Handling, 14(3), 541-550 (1994)

Cabrejos, F., M.I. Jofré y J. Rojas, Transporte Neumático de Materiales Sólidos a Granel, Actas Congreso CONAMET/SAM, La Serena, Chile, 3 al 4 de Noviembre (2004)

Deng,T. y M.S. Bradley, Determination of a Particle Size Distribution Criterion for Predicting Dense Phase Pneumatic Conveying Behaviour of Granular and Powder Materials, Powder Technology, 304, 32-40 (2016)

Gómes, L.M. y A.L. Amarante Mesquita, On the Prediction of Pickup and Saltation Velocities in Pneumatic Conveying, Brazilian Journal of Chemical Engineering, 31(01), 35-46 (2014)

Hartman, M., M. Pohořelŷ y O. Trnka, Transport Velocities of Different Particulate Materials in Pneumatic Conveying, Chem. Pap., Institute of Chemistry, Slovak Academy of Sciences, 60(1), 74-77 (2006)

Hubert, M. y H. Kalman, Experimental Determination of Length-Dependent Saltation Velocity in Dilute Flows, Powder Technology, 134 (1), 156-166 (2003)

Jo, Y.M. y M.B. Ray, Pneumatic Transportation of Powder in Horizontal Pipelines, Journal of Industrial and Engineering Chemistry, 5(1), 32-39 (1999)

Jones, P.J. y L. S. Leung, Estimation of Saltation Velocity in Horizontal Pneumatic Conveying. - A Comparison of Published Correlation, Pneumotransport, $4^{\text {th }}$ International Conference on the Pneumatic Transport of Solids in Pipes, California, USA, June 26-28, C1-1-C1-12 (1978)

Khan, T.S., Y. Dai, M.S. Alshehhi y L. Khezzar, Experimental Flow Characterization of Sand Particles for Pneuamtic Transport in Horizontal Circular Pipes, Powder Technology, 292(1), 158-168 (2016)

Leva, M., Fluidization, McGraw-Hill, New York, USA (1959)

Matsumoto, S., M. Kara, S. Saito y S. Maeda, Minumum Transport Velocity for Horizontal Pneumatic Conveying, Journal of Chemical Engineering of Japan, 7(6), 425-430 (1974)

Matsumoto, S., M. Harada, S. Saito y S. Maeda, Saltation Velocity for Horizontal Pneumatic Conveying, Journal of Chemical Engineering of Japan, 8(4), 331-333 (1975)

Molerus, O. y U. Heucke, Pneumatic Transport of Coarse Grained Particles in Horizontal Pipes, Powder Technology, 102, 135-150 (1999)

Patankar, A., Makawana, A. y M. Bose, Lectura Notes in Mechanical Engineering, F8, 1609-1616 (2017)

Pocoví, R.E., Ventilación Industrial, 1ª. Ed., Ediciones Magna Publicaciones, Salta, Argentina (1999)

Pocoví, R., J. Flores y G. Villaflor, Estudio de la Operación de las Campanas de Aspiración de Polvos en Sistemas de Ventilación Localizada, Información Tecnológica (CIT), 9 (2), 139-146 (1998)

Pocoví, R., J. Flores, G. Villaflor, J. Velasco, A. Redondo y E. Bisonard, Diseño de Ciclones para Instalaciones de Ventilación Localizada. Criterio para Maximizar la Eficiencia de Separación, Innovación, Universidad de Antofagasta, Chile, 1(14), 25-34 (2002)

Rabinovich, E. y H. Kalman, Threshold Velocities of Particle-Fluid Flows in Horizontal Pipes and Ducts: Literature Review, Chemical Engineering, 27 (1), 215-239 (2011)

Setia, G., S.S. Mallick, R. Panb y P.W. Wypychc, Modeling Minimum Transport Boundary for Fluidized DensePhase Pneumatic Conveying Systems, Powder Technology, 277, 244-251 (2015) 